\title{
A Numerical Approach to Investigate the Influence of Resonator Setting and Volume Fraction on Stop Bands in an Acoustic Metamaterial
}

\author{
Riaz Ahmed ${ }^{l, *}$, Hossain Ahmed ${ }^{2}$ and Sourav Banerjee ${ }^{2}$ \\ ${ }^{1}$ University of Wisconsin - Green Bay, Green Bay, WI 54311, USA \\ ${ }^{2}$ University of South Carolina, Columbia, SC 29208, USA
}

Received: September 23, 2020, Revised: September 28, 2020, Accepted: September 29, 2020, Available Online: October 03, 2020

\begin{abstract}
A quest for physics-based understanding of engineered metamaterials inspired numerous researchers to extract intricate features such as guiding and filtering elastic waves, wave focusing, topological insulation, cloaking etc. A traditional metamaterial is composed of a heavy core along with some other materials of dissimilar mechanical properties (e.g. stiffness, density). It is well-established that the frequency band gap can be formed by introducing the desired inconsistency of material properties between stiffer resonators and adjacent embedding matrices. Frequency band gaps are fundamental requirements of many engineering applications such as vibration control, noise mitigation, and energy concentrations. Hence, advanced researches are being carried out continuously to understand the control parameters (e.g. bandwidth, starting and ending frequencies) of the frequency band gap. In this article, a mass-in-mass metamaterial using elliptical anisotropic resonators are considered to investigate the influence of resonators' geometric factors and volume fraction on band gap parameters. While the elliptical resonator is splitted diagonally in one or both opposite ends to analyze the influence of volume fraction of the resonators on stop bands, a second set of half-circular resonators are also investigated to analyze the impact of resonator parameters on frequency bands.
\end{abstract}

Keywords: Acoustic metamaterial; Band gap; Split-ring resonator; Volume fraction; Wave filtration.

This work is licensed under a Creative Commons Attribution-Non Commercial 4.0 International

\section{Introduction}

From the last couple of decades, electromagnetic metamaterials [1],[2] are in concentration to phononic researchers to explore photonic band gaps, a range of frequency where electromagnetic waves cannot propagate [3]. The concept of electrodynamics metamaterial can be used in designing elastrodynamic metamaterials since the physical understanding of electromagnetic wave propagation in photonic crystals is quite similar to the stress (elastic) wave propagation in phononic crystals [4],[5]. Mass-in-mass systems are frequently proposed under elastodynamic problems to predicatively manipulate the frequency band gaps in metamaterials with engineered volume inclusions [6],[7]. Creating local resonance vibration modes is one of the important features of such acoustic metamaterials. Applications of such acoustic metamaterials have been envisioned for waveguiding, vibration control, sound isolation, sensing, energy harvesting etc. [8]-[12].

Band gap and band structure manipulation are increasingly important in acoustic metamaterials to branch out its applicability. It is well-established that in any metamaterial frequency band gap are results from either local resonance or Bragg scattering. Low-frequency sound can be controlled by introducing locally resonant components into phononic crystals [13]-[17], whereas conventionally high-frequency stop bands can be formed by multiple scattering (Bragg) of the periodic inclusion [18],[19]. In recent years, researchers are attempting to manipulate the ability of the metamaterial by artificially designing the system. It has been proven that the number of stop bands can be increased by adding additional local resonators in the system [20],[21]. Most recently a multi-scale mass-in-mass model (MMM) with split-ring resonators [22] is proposed not only to obtain a wider frequency band but also to obtain multiple band gaps in both sonic and ultrasonic frequency regions. The concept of using split rings in a metamaterial system has primarily emerged in photonics research. Movchan et al. [23] used split-ring resonators to control electromagnetic bands in two-dimensional photonic structures. Many other researchers in photonic domain found split ring useful in manipulating electromagnetic waves for specific purposes.

Inspired from these researches, a symmetric split-ring metamaterial system with multiple resonators is considered herein as the model offers a high level of anisotropy. Note that, anisotropy in a unit cell is one of the key factors of dispersive behavior and band gap formation in a mass-in-mass metamaterial system. Although the size effect of the resonators on the respective band gaps was reported earlier [24], the geometric (shape)/volumetric dependency of the resonators on frequency bands is still to explore. Hence, the primary objective of this study is to investigate the possibility to systematically manipulate the frequency band gap by the alteration of the resonator's geometry or volume fraction. In addition, two different sets of resonators in a unit cell are also investigated to understand the influence of resonator setting on the frequency band gap.

\section{Model and Computational Approach}

\subsection{Model Configurations}

Several two-dimensional structures with multiple resonator systems (Ref. Fig. 1) are designed to understand the geometric/volumetric dependency of the local resonators on frequency bands. Initially, the unit cell is composed of a heavy core (R1) of diameter 0.1414 inches embedded in a circular ring 
(R2) with an outside diameter of 0.2828 inches. A softer material (M1), say, Rubber, is used to seal the space between the heavy core and the circular ring. A similar mass-in-mass system was also proposed by Huang et al. [21]. However, an elliptical ring (R3) is placed at the center of the unit cell to further enhance the anisotropic property of the system (Fig. 1 (a)). The elliptical ring is rotated at 450 about the center of the unit cell to allow identical wave pathway along both $\mathrm{x}$ - and $\mathrm{y}$-directions. A much stiffer epoxy material M2 compared to M1 is considered to fill in the elliptical free space. In order to allow continuous mismatch in material stiffness between components in the system, a polymer material (M3) is considered to complete the outer boundary of the unit cell. The outside dimension of the unit cell is measured as one (1) square inch.
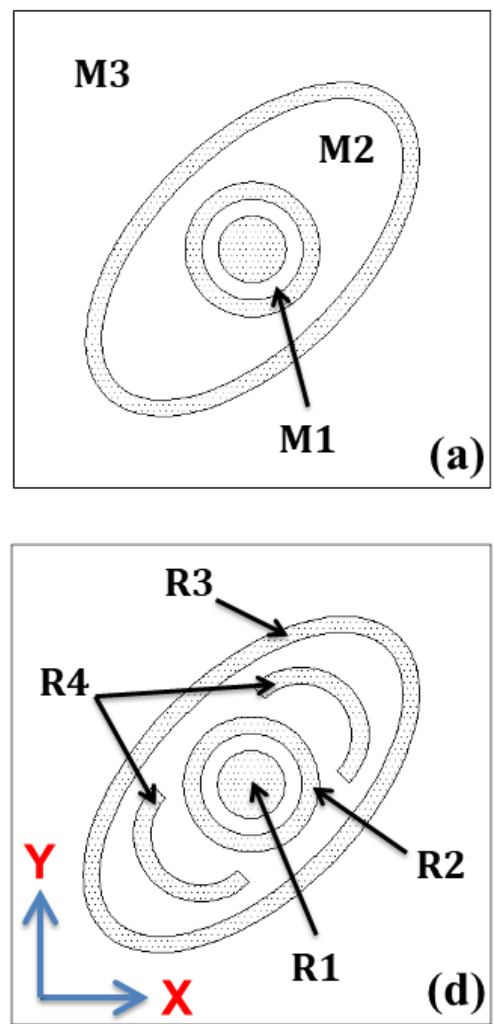

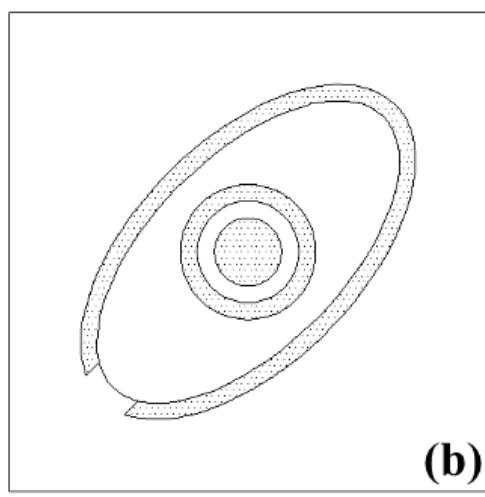

(b)

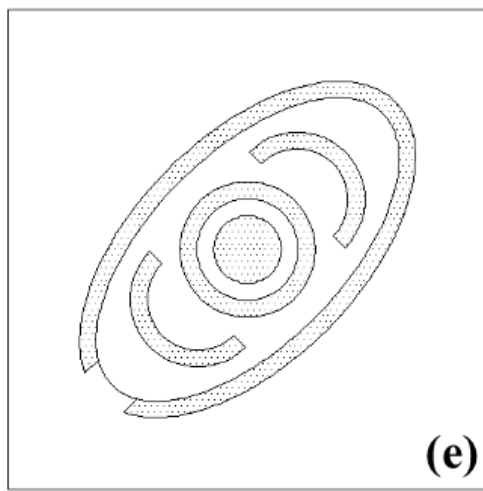

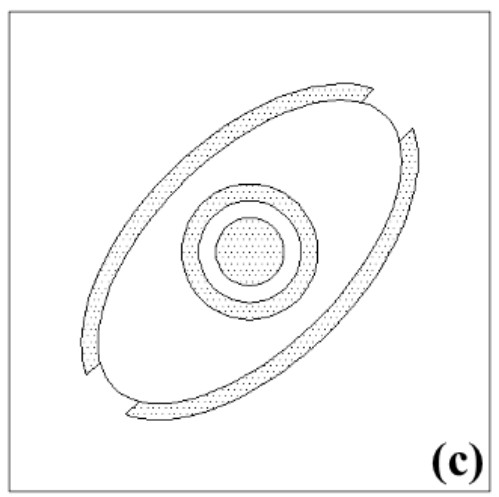

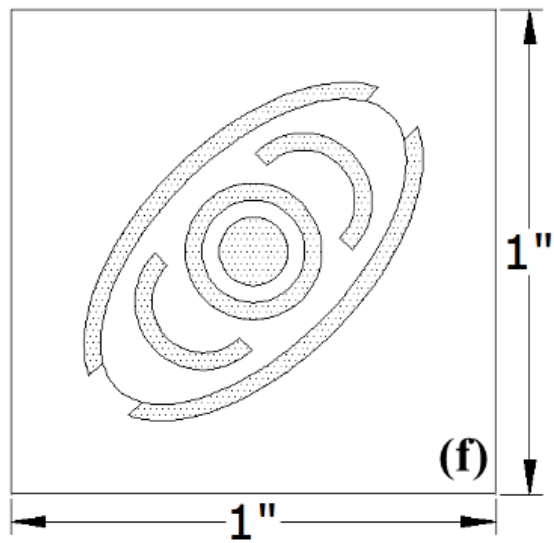

Fig. 1 Projected models to analyze the geometric influence of local resonators on frequency bands. Models (1a-1c) with resonators R1-R3 are considered as set-1 and rest (1d-1f) as set-2 with R1-R4 resonators.

While designing, the elliptical resonator is splitted about 0.115 inches along its major axis in both one (Fig. 1(b)) and two (Fig. 1(c)) directions, respectively. Such splitting of the resonators not only decreases the volume fraction of the resonators in the unit cell but it also allows additional degrees of freedom in the system, which tends to alter the frequency bands. The splitting of the resonators was made at the ends of the major axis of the elliptical geometry to ensure similar wave propagation path along $\mathrm{x}$ - and $\mathrm{y}$ - directions. In order to analyze the geometric

Table 1 Properties of the components of the unit cell. influence of the resonators more rigorously, especially the effect of resonator setting, a pair of half-circular rings (R4) are added symmetrically (Figs. 1(d)-1(e)) in all three models described previously (Figs. 1(a)-1(c)). Unit cells with resonators R1-R3 are considered as resonator set-1 (Models 1a-1c); whereas set-2 is referred to as models with resonators R1-R4 (Models 1d-1f). In this study, the thickness of all the rings is considered as 0.037 inches. Material properties and dimensions of the unit cell components are listed in Table 1.

\begin{tabular}{|c|c|c|c|c|}
\hline $\begin{array}{l}\text { Component } \\
\text { Name } \\
\end{array}$ & $\begin{array}{c}\begin{array}{c}\text { Outer Dimensions } \\
\text { (inch) }\end{array} \\
\end{array}$ & $\begin{array}{l}\text { Stiffness } \\
(\mathbf{P a})\end{array}$ & $\begin{array}{l}\text { Density } \\
\left(\mathrm{kg} / \mathrm{m}^{3}\right)\end{array}$ & $\begin{array}{l}\text { Poisson's } \\
\text { Ratio } \\
\end{array}$ \\
\hline$M 1$ & Diameter -0.2121 & $10 \mathrm{e} 6$ & 980 & 0.49 \\
\hline$M 2$ & $\begin{array}{l}\text { Major Radius }-0.4 \\
\text { Minor Radius }-0.2\end{array}$ & $2.5 \mathrm{e} 9$ & 1250 & 0.38 \\
\hline$M 3$ & $1 \mathrm{X} 1$ Square & $0.5 \mathrm{e} 9$ & 1050 & 0.49 \\
\hline$R 1$ & Diameter -0.1414 & $13 \mathrm{e} 9$ & 11310 & 0.435 \\
\hline$R 2, R 4$ & Diameter -0.2828 & $100 \mathrm{e} 9$ & 2950 & 0.31 \\
\hline$R 3$ & $\begin{array}{l}\text { Major Radius }-0.435 \\
\text { Minor Radius }-0.235\end{array}$ & $100 \mathrm{e} 9$ & 2950 & 0.31 \\
\hline
\end{tabular}




\subsection{Numerical Simulation}

The mathematical formulation of infinitely repeating split ring unit cell proposed in this study requires the implementation of Bloch theorem. Since it is challenging to perform analytical derivation of such a material system, the widely accepted Finite Element Method has been utilized to solve this problem. While calculating the dispersion relation, the complete structure is considered infinite in both $\mathrm{x}$ - and $\mathrm{y}$ - directions by arranging the unit cells or the Representative Volume Elements (RVEs) periodically. To implement this concept, the Bloch-Floquet periodic boundary condition is applied at all boundaries of the RVE. These boundary conditions are based on the Floquet theory that can be applied to the problem of vibrations with smallamplitude in spatially periodic structures. The theory states that the solution can be sought in the form of a product of two functions. One follows the periodicity of the structure, while the other one follows the periodicity of the excitation. The problem can be solved on a unit cell of periodicity by applying the corresponding periodicity conditions to each of the two components in the product. Generalized wave equation in the composite material can be written as

$$
\begin{gathered}
C_{i j k l}\left(x_{m}\right)\left[u_{k, l}\left(x_{m}, t\right)+u_{l, k}\left(x_{m}, t\right)\right]+f_{i}\left(x_{m}\right) \\
=\rho\left(x_{m}\right) \ddot{u}_{l}\left(x_{m}, t\right)
\end{gathered}
$$

where the constitutive matrix containing material properties and the density of the system are the functions of space $\left(x_{1}, x_{2}\right)$. $i, j, k, l \& m$ takes values $1,2 \& 3$. Standard index notation is used throughout this manuscript. Let the body force $f\left(x_{m}\right)$ be constant.

The unit cells are repeated in both directions and the solution can be assumed in terms of Bloch-Floquet solution as discussed in the previous paragraph. Assuming there is no periodicity along $x_{3}$ direction \& decoupling the phase component, the displacement solution can be viz.

$$
\begin{aligned}
& u_{i}\left(x_{m}, t\right)=\sum_{n 2} \sum_{n 1} A_{n 1 n 2}^{i} \exp \left(i k_{m} x_{m}\right) . \\
& \exp \left(i G_{m} x_{m}\right) \cdot \exp \left(i k_{3} x_{3}\right) \cdot \exp (-i \omega t)
\end{aligned}
$$

Where, $k_{m}$ is wave number along $m$-th direction and $G_{m}$ is a component of the reciprocal lattice vector along $m$-th direction. Here, $m$ takes values $1 \& 2$. $G_{m}$ can be expressed as $G_{m}=$ $2 \pi n_{m} / D_{m}$, where, $D_{m}$ is the periodicity of the cells in $m$-th direction. The $A^{i}{ }_{n 1 n 2}$ is the amplitude of the wave modes for particle displacement along $i$ and $n_{1} \& n_{2}$ are the integer numbers between $-\infty$ to $+\infty$. After substituting Eq. (2) in Eq. (1) the Bloch equation with the Bloch operator can be obtained as follows

$$
\begin{aligned}
& \omega^{2} \rho\left(x_{m}\right) \sum_{n 2} \sum_{n 1} A_{n 1 n 2}^{i} \exp \left(i\left(k_{m}+G_{m}\right) x_{m}\right. \\
& -\frac{1}{2} C_{i j k l}\left(x_{m}\right)\left(k_{m}\right. \\
& \left.\left.+G_{m}\right)^{2} \delta_{m j}\right)\left[\sum_{n 1} \sum_{n 2} A_{n 1 n 2}^{k} \exp \left(i\left(k_{l}+G_{l}\right) x_{l}\right)\right. \\
& \left.+\sum_{n 1} \sum_{n 1} A_{n 1 n 2}^{l} \exp \left(i\left(k_{k}+G_{k}\right) x_{k}\right)\right]=0
\end{aligned}
$$

The above equation is a Bloch eigenvalue problem. The Eq. (3) is then multiplied with Bloch operator with Bloch transformed weighting factor and integrated over the whole domain of the body and the equation was transformed to its weak form. Periodic boundary conditions are applied around the unit cell and weak form of Bloch equation is solved only within the irreducible Brillouin Zone. Further number of amplitude in the Eq. (3) is reduced for each wavenumber (k) point. Thus the $n_{1} \& n_{2}$ are reduced from infinity and the truncated set of Bloch mode expansions were used in the solution method. A suitable choice of reduced-order basis was made based on the high symmetry points that characterize the periodic lattice. Next, the Finite Element discretization was performed. Triangular isoparametric elements were used in the simulation. Element sizes were determined based on a series of convergence study and the minimum wavelength occurred in the material. The sizes of the elements were kept to a minimum of $1 / 10$ of the corresponding minimum wavelength that occurred in any material type, respectively. The Bloch displacement amplitudes were discretized using isoparametric shape function $\left(N_{i}(\boldsymbol{x})\right)$ suitable for triangular elements for each combination of $n_{1} \& n_{2}$ in their truncated series as follows

$$
\boldsymbol{A}_{n 1 n 2}=\sum_{i=1}^{3} N_{i}(\boldsymbol{x}) \Lambda_{i}
$$

Applying the discretization equations and periodic boundary conditions the weak form of Bloch equation reduces to an algebraic eigenvalue problem $\left[\mathbf{K}(k)-\omega^{2} \mathbf{M}\right] \widetilde{\mathbf{V}}=\mathbf{0}$, where $\widetilde{\mathbf{V}}$ is the discrete Bloch amplitude vector which is periodic within the unit cell. The $\mathbf{K}(k)$ and $\mathbf{M}$ are the global stiffness and mass matrices, respectively obtained by integrating the element level matrices in proper order. Detail expressions for $\mathbf{K}$ and $\mathbf{M}$ can be found in reference. The solution of the eigenvalue problem provided the dispersion curves for the proposed periodic media.

\subsection{Validation of Computational Approach}

The studied model configurations in this work are quite complex. Hence, it is extremely challenging to perform the mathematical formulation and analytically solve the proposed models. Hence, a simple mass-in-mass system (Ref. Fig. 2) is studied herein using the Finite Element tool to validate the solution methodology.

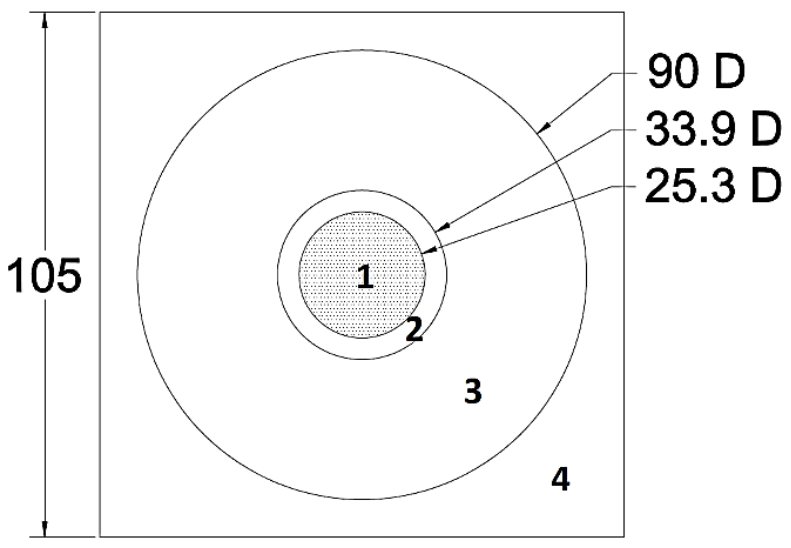

Fig. 2 Single-resonator mass-in-mass system with square periodicity (dimensions are in $\mathrm{mm}$ ). 
In this simple system, a unit cell is designed which is composed of four components. A significantly stiff material, lead (component 1), is chosen as a circular resonator [4],[25]. To avoid evanescent wave modes, the core resonator is coated with softer rubber-like material (component 2). The rubber-lead arrangement is then embedded into a relatively stiffer component (epoxy). The lead-rubber-epoxy combination is synonymous with a well-known mass-in-mass engineered material system proposed by the earlier researchers [20]. It is mathematically proven that such an engineered system provides frequency band gaps (stop bands) by virtually creating negative bulk modulus and negative mass density in the structure [21],[26]. In engineered material systems, the negative responses result from the mismatch of material properties between adjacent components. Hence, to induce further property mismatch, the mass-in-mass system is placed inside a square unit element and the created gap is filled with a relatively softer material (polyethylene plastic).

The complete lead-rubber-epoxy-polyethylene design is considered as the Representative Volume Element (RVE) in this section. The unit cell is a $105 \mathrm{~mm}$ square and diameters of the lead, rubber, and epoxy components are considered as $25.4 \mathrm{~mm}$, $33.9 \mathrm{~mm}$, and $90 \mathrm{~mm}$, respectively. The material property of the unit cell components is listed in Table 2.

Table 2 Properties of the components enclosed in the unit cell.

\begin{tabular}{ccccc}
\hline & Lead & Rubber & Epoxy & Polyethylene \\
\hline $\begin{array}{c}\text { Young's } \\
\text { Modulus (Pa) } \\
\text { Density } \\
\left(\mathrm{kg} / \mathrm{m}^{3}\right)\end{array}$ & $13 \mathrm{e} 9$ & $10 \mathrm{e} 6$ & $3.5 \mathrm{e} 9$ & $0.7 \mathrm{e} 9$ \\
$\begin{array}{c}\text { Poisson's } \\
\text { Ratio }\end{array}$ & 0.44 & 0.49 & 0.38 & 0.49 \\
\hline
\end{tabular}

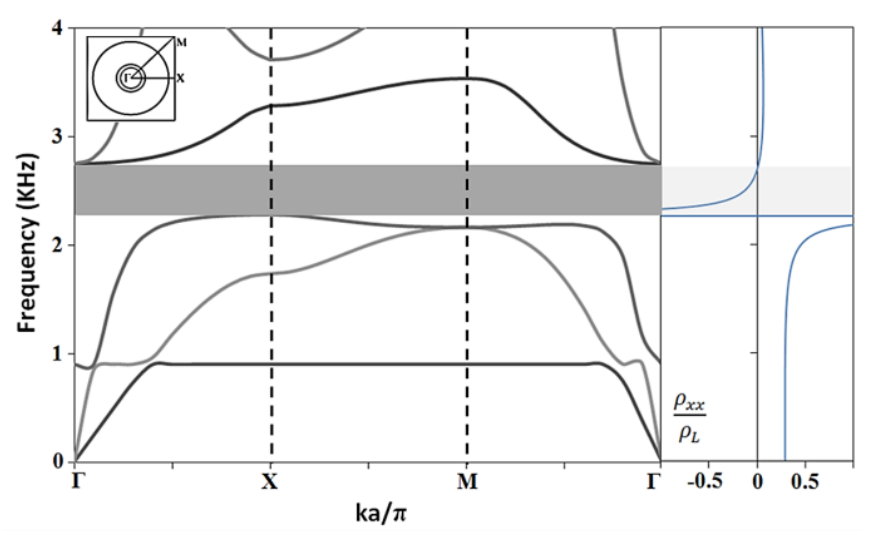

(a)

(b)

Fig. 3 (a) Dispersion relation (b) Mass density plot for the single-resonator mass-in-mass system.

It is well known that the frequency band gaps are the result of negative mass density and induction of resonance in local microstructures of metamaterials [20],[21],[14],[24]. To validate the above statement and adopted FEM solution methodology, a multi-layered mass-in-mass model (Ref. Fig. 2) is considered. This study is carried out for a frequency range of 0 to $10 \mathrm{KHz}$. For illustration purposes, the response is reported between 0 to 4 $\mathrm{KHz}$ in Fig. 3. Computed effective mass densities are normalized by the density of the stiffest component (lead) of the cell. The effective mass density of the unit cell is calculated by Eq. (5) using long wavelength assumption.

$$
\rho_{\alpha \beta}=\frac{\int \sigma_{\alpha \beta} d \Gamma}{\int \ddot{u}_{\alpha \beta} d \Gamma}
$$

where $\alpha, \beta=1,2$ and $\sigma_{\alpha \beta}$ and $\ddot{u}_{\alpha \beta}$ are the local stress and acceleration quantities. $\Gamma$ denotes the external boundary of the unit cell.

The dispersion curve for the single resonator metamaterial within the first Brillouin zone is shown in Fig. 3(a). Using the definition of mass density in Eq. (1), it has been found that the mass density of the system stays negative between the frequency range of $2.27-2.71 \mathrm{KHz}$ (Ref. Fig. 3(b)). Alternatively, a stop band is observed (Ref. Fig. 3(a)) at a frequency range between $2.28-2.74 \mathrm{kHz}$ (considering $\Gamma-\mathrm{X}$ directional waves). The same band gap continues for the entire $\Gamma-\mathrm{X}-\mathrm{M}-\Gamma$ directional waves. Since the mass density of the metamaterial (calculated using Eq. (1)) is found negative within the frequency range of the stop band, it can be concluded that the analytical and numerical outcomes are in close agreement. This phenomenon is well reported in the literature [20],[21],[14],[24],[27],[28].

\section{Results and Discussion}

In order to investigate the influence of resonator geometry, six (6) models are proposed as described in Fig. 1. It is hypothesized that resonator geometry as well as the volume fraction of resonators in the unit cell and type of resonator arrangement may have a considerable impact on frequency bands. The dispersion relation of the irreducible Brillouin zone (see Fig. 4(b)) for model 1a (Ref. Fig. 1(a)) is shown in Fig. 4(a). Note that, the wave vector is normalized by the length of the unit cell 'a'.

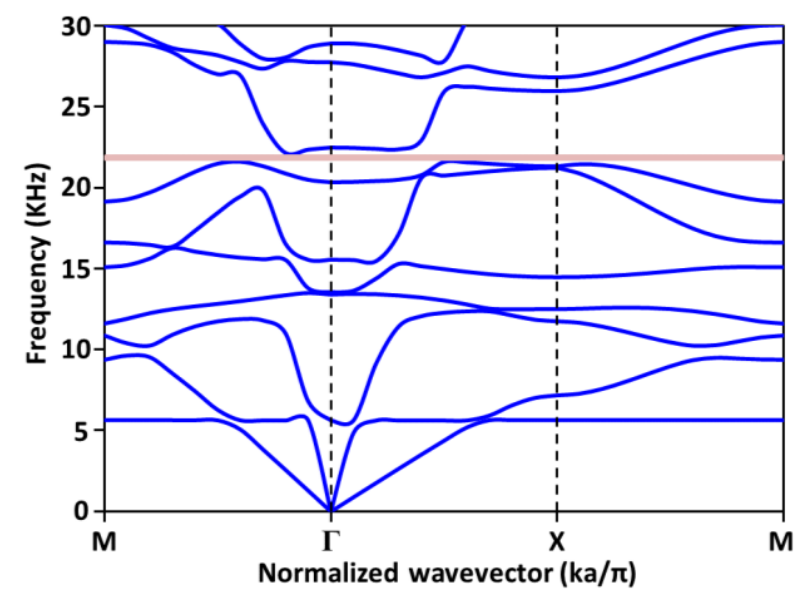

(a)

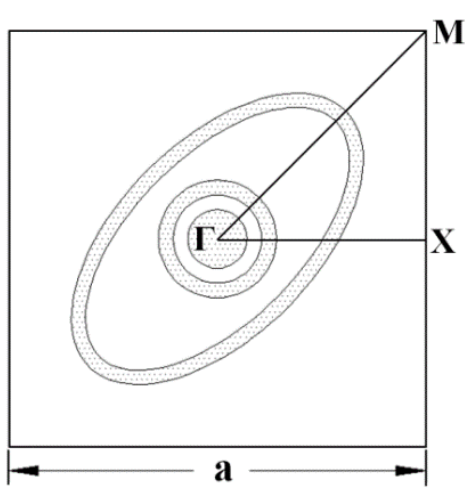


(b)

Fig. 4 (a) Dispersion curve and band gap representation for model 1a. (b) First Brillouin Zone.

In Fig. 4(a), almost all the frequency bands seem highly dispersive, whereas a strong weakly-dispersive (straight) region is observed in the first frequency band, and a stop band is noticed

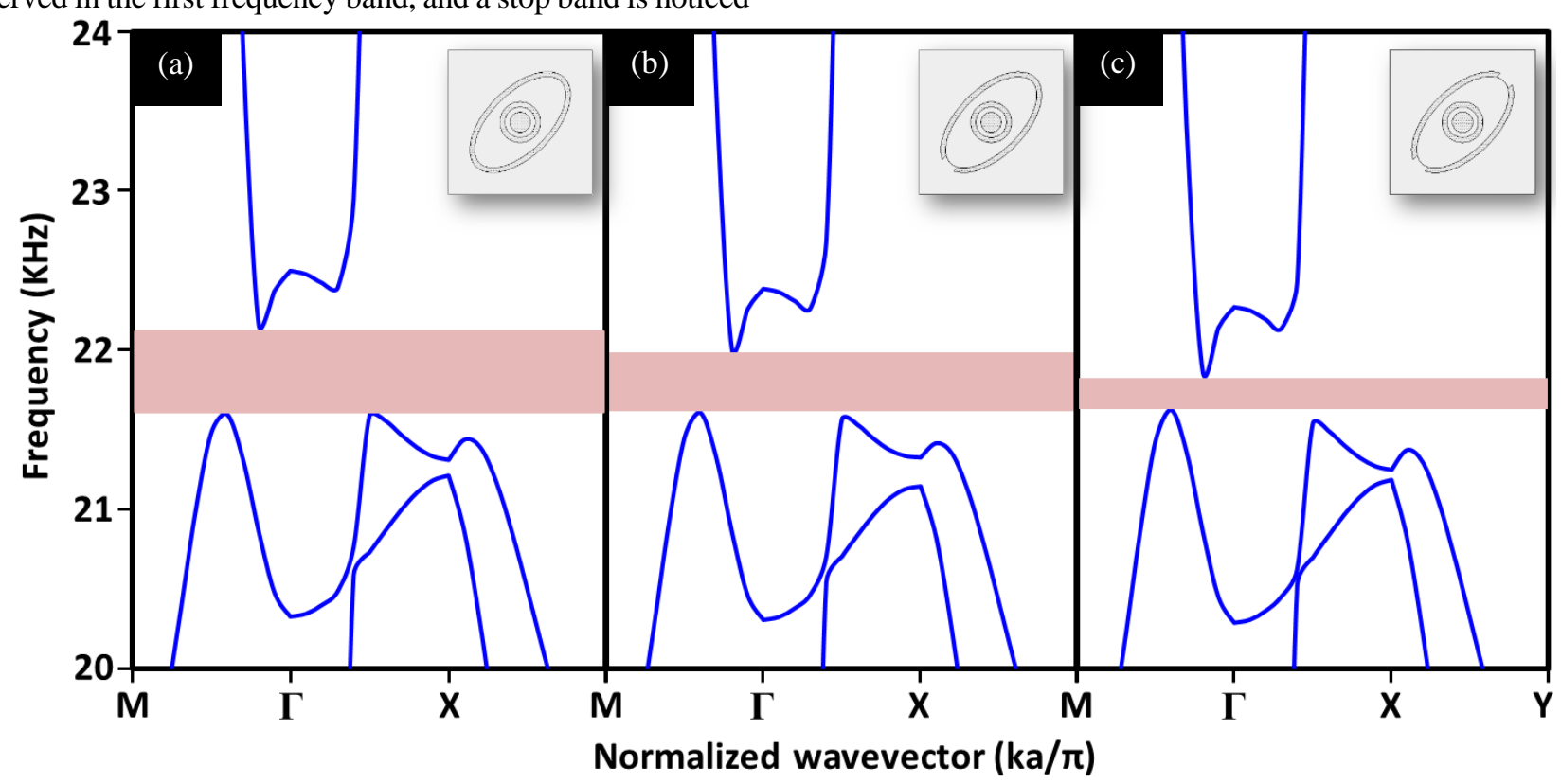

Fig. 5 Magnified dispersion curve representations for resonator set-1 (models 1a-1c).

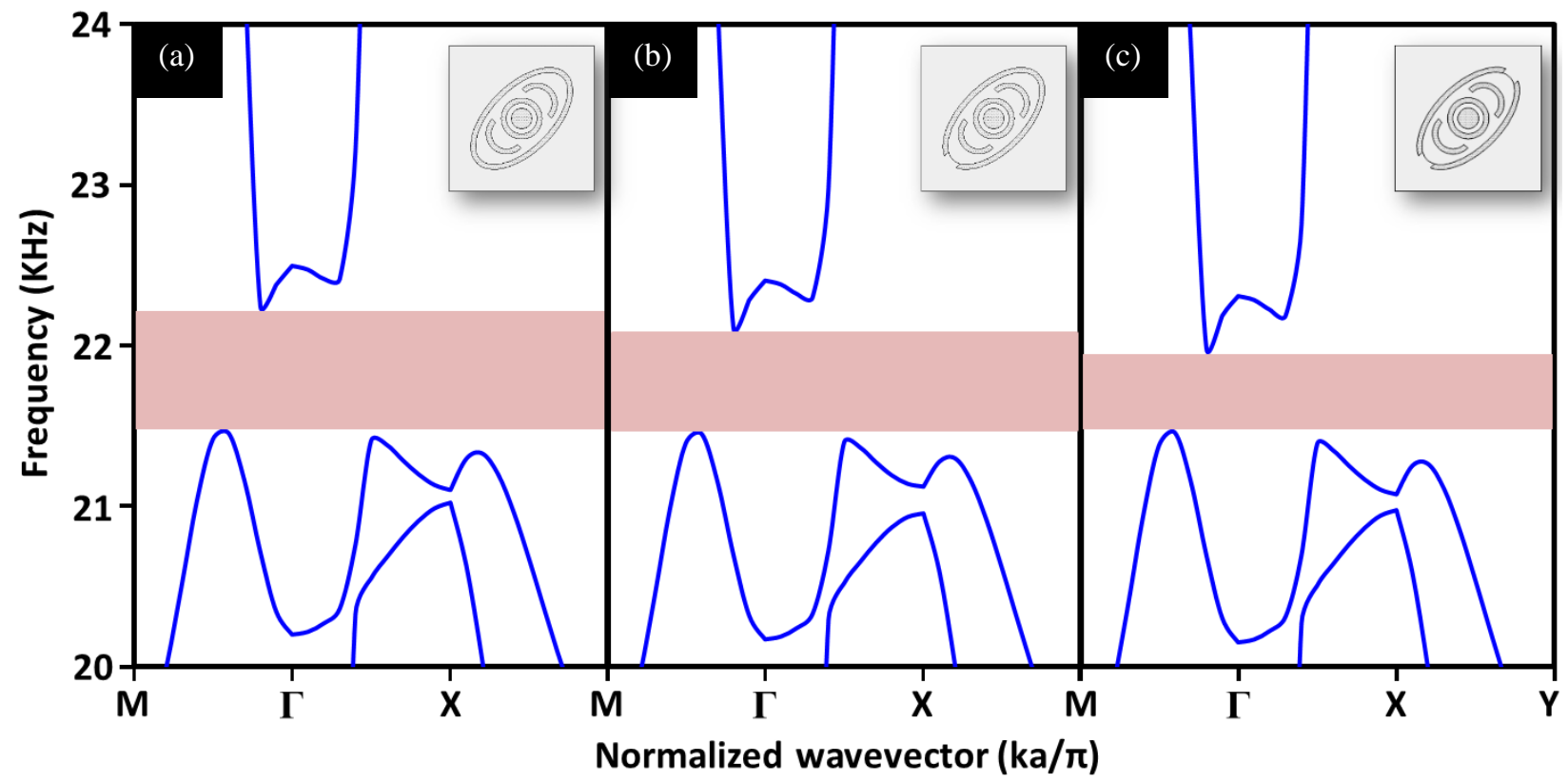

Fig. 6 Magnified dispersion curve representations for resonator set-2 (models 1d-1f).

A band gap with a bandwidth of $562 \mathrm{~Hz}$ is observed between $21.598 \mathrm{KHz}$ to $22.16 \mathrm{KHz}$ for the unit cell with a center ball, circular ring, and closed elliptical ring resonators (Fig. 5(a)) and Table 3). However, the bandwidth is reduced to $402 \mathrm{~Hz}$ (Fig. 5(b)) and $241 \mathrm{~Hz}$ (Fig. 3(c) and Table 3) through the splitting of the elliptical resonator in one end and both ends, respectively. A similar trend is also noticed for other sets of models where two additional half-circular resonators are placed symmetrically. The longest stop band is recorded for model 1d with a bandwidth of between $7^{\text {th }}$ and $8^{\text {th }}$ frequency bands. The primary focus in this section is to study the transformation of the band gaps due to the alteration of resonator geometry and settings, For comparison, the magnified region of the band gaps for all the models are plotted in Fig. 5 (set 1) and 6 (set 2). 
different volume fractions of the resonators and there is also a possibility to obtain longer band gaps with unchanged volume fraction. In both cases, the selection of resonators configuration in the unit cell would be the key challenge.

Table 3 Obtained band gaps and volume fraction of resonators in the unit cell for studied models.

\begin{tabular}{ccccc}
\hline Model \# & $\begin{array}{c}\text { Band Start } \\
(\mathbf{H z})\end{array}$ & $\begin{array}{c}\text { Band End } \\
(\mathbf{H z})\end{array}$ & $\begin{array}{c}\text { Band Gap } \\
(\mathbf{H z})\end{array}$ & $\begin{array}{c}\text { Volume Fraction } \\
(\boldsymbol{\%})\end{array}$ \\
\hline$a$ & 21598 & 22160 & 562 & 11.38 \\
\hline$b$ & 21608 & 22010 & 402 & 11.17 \\
\hline$c$ & 21623 & 21864 & 241 & 10.96 \\
\hline$d$ & 21454 & 22246 & 792 & 14.13 \\
\hline$e$ & 21453 & 22114 & 661 & 13.91 \\
\hline$f$ & 21457 & 21985 & 528 & 13.70 \\
\hline
\end{tabular}

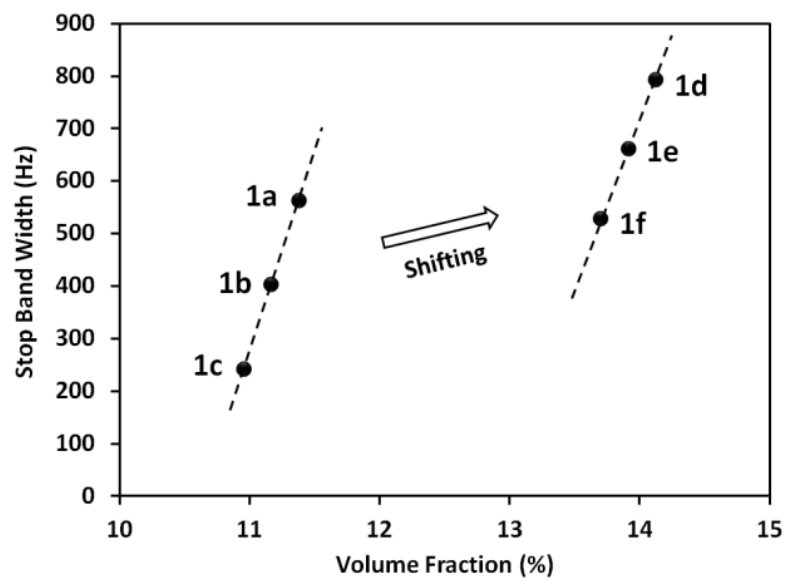

Fig. 7 Stop band manipulation through the alteration of resonators volume fractions and setting in unit cell. Each bullet defines the obtained stop bandwidth with respect to the volume fraction of the resonators in the corresponding proposed model (1a-1f, see Fig. 1).

Fig. 7 shows the influence of the volume fraction of the resonators on OVERALL stop bandwidth. Here, OVERALL means, these stop bands can be found for wave propagation through the model along any direction. While the overall band gap of a structure is important for filtering wave propagation in a three-dimensional space, guided wave propagation (along a specific direction) in a structure is not uncommon. Fig. 8(a) represents the stop bandwidths for both set- 1 and set- 2 if the wave enters the structure as a guided $\mathrm{x}$-directional $(\Gamma-\mathrm{X})$ wave. Fig. 8(b) represents a similar phenomenon in the case of the $y-$ directional (X-M) wave. It can be seen that the stop bands are heavily influenced by the volume fraction of the resonator for wave propagation in $\Gamma$-X direction, which is similar to the overall response reported in Fig. 7 However, in the case of wave propagation in X-M direction, the effect of volume fraction isn't much significant, where a little increase in stop bandwidth is identified (Fig. 8(b)).

In a bandgap region, it can be seen that two bands from the dispersion curve take a role in forming the stop band (Ref. Fig. 4(a)). To understand the influence of resonator volume fraction on those band gap forming frequency bands, a further investigation is performed and the results are presented in Figs. 9-11. Fig. 9 presents the shift in the lower and upper band of the overall bandgap for different volume fractions. Figs. 10 and 11 present similar outputs for $\mathrm{x}$-directional and $\mathrm{y}$-directional guided waves, respectively. In all these figures, it can be noticed that the volume fraction of the resonators in the unit cell possesses almost no influence on the lower band (start frequency) of the stop band for both sets (a-c and d-f) of models.

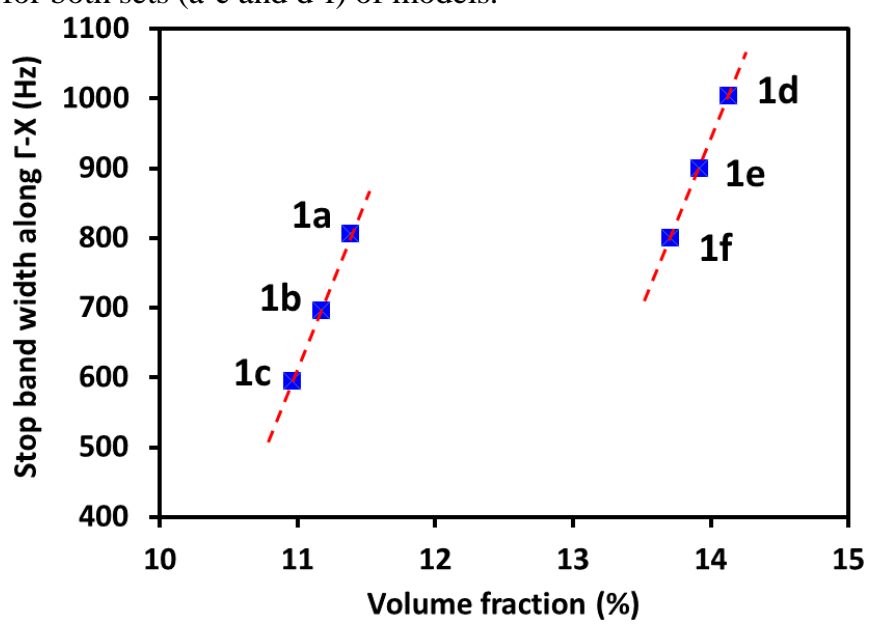

(a)

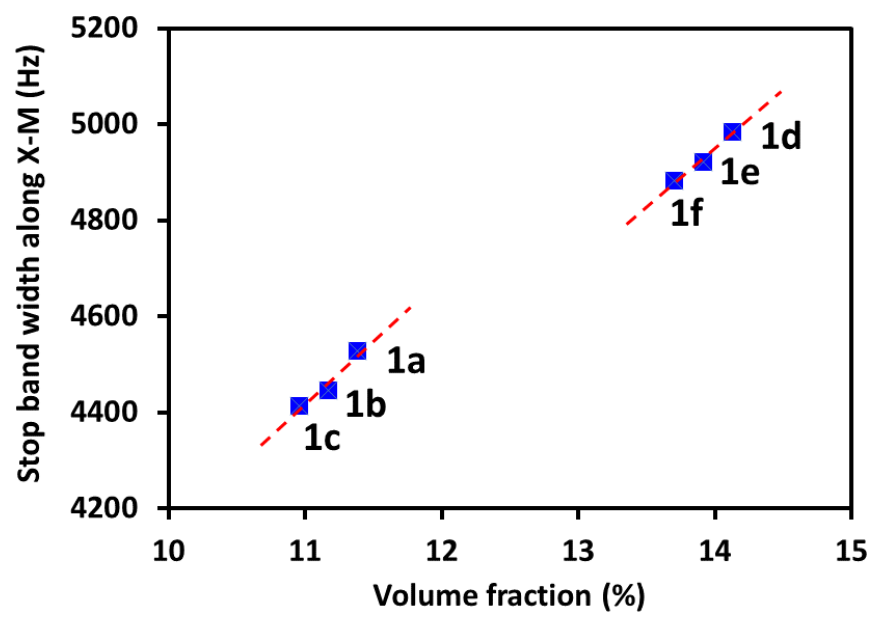

(b)

Fig. 8 Influence of volume fraction on stop bandwidth for wave propagation along (a) $\Gamma-\mathrm{X}$ or $\mathrm{X}$-direction, and (b) $\mathrm{X}-\mathrm{M}$ or $\mathrm{y}$-direction.

However, the upper bands of the bandgap show a significant dependency on the volume fraction. Note that, for X-M 
directional wave incidence (Fig. 11) both the upper and lower band of the band gap show very little reliance on volume fraction. Henceforth, the bandgap width also remains almost unchanged. Upon investigation of the mode shape, it has been found that the frequency bands forming the stop bands along X-M direction are predominantly the result of Bragg scattering, where deformation of the matrix element in the unit cell controls the formation of the band structure. In such instances deformation of the ring resonators is quite negligible. Hence, a change in the volume

Models a-c

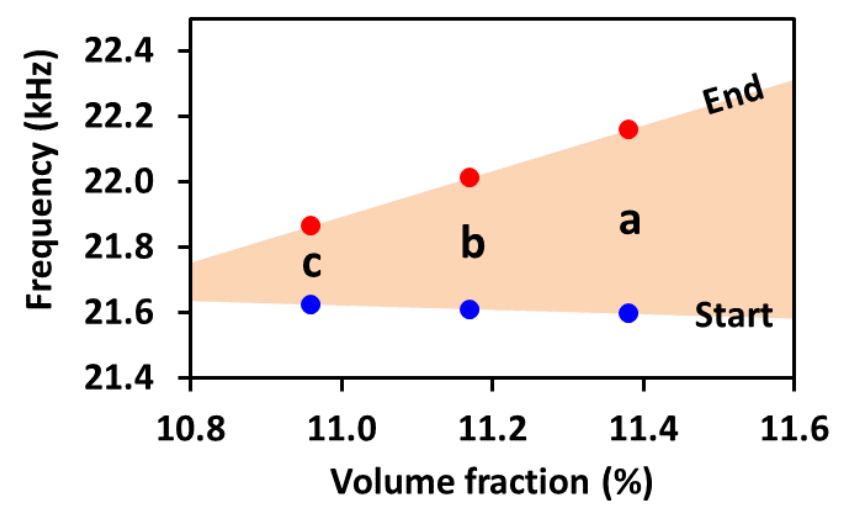

(a) fraction of the ring resonators making a negligible effect on the band structure or band gap along X-M direction. On the contrary, the upper band to form the frequency band gap along $\Gamma-X$ direction is the result of the combined effect of Bragg scattering and local resonance of the metal resonators. Such observation is also true for the upper bands of the overall stop bands in Fig. 9. Hence, a change in the volume fraction of the resonators shifted the upper bands for both overall and $\Gamma$-X directional waves.

\section{Models d-f}

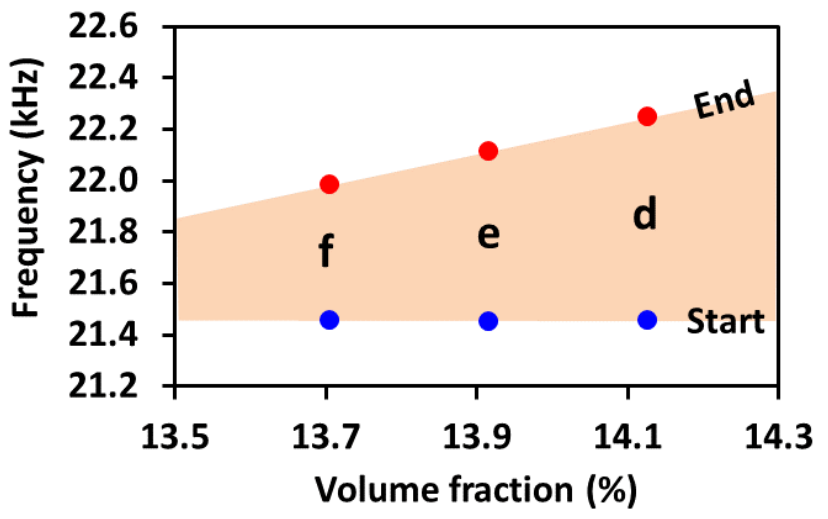

(b)

Fig. 9 Shift in start and end of the overall band gap due to the change in volume fraction

Models a-c

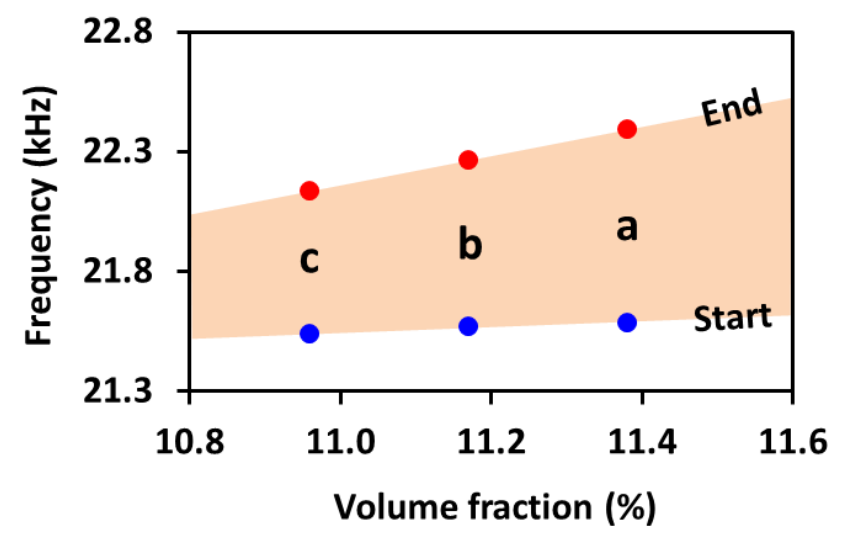

(a)
Models d-f

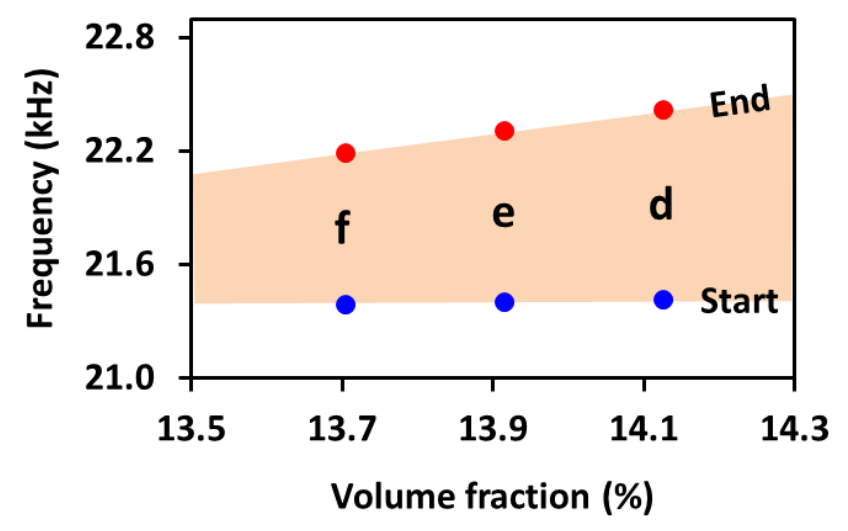

(b)

Fig. 10 Shift in start and end of the band gap along $\Gamma-X$ due to the change in volume fraction

Models a-c

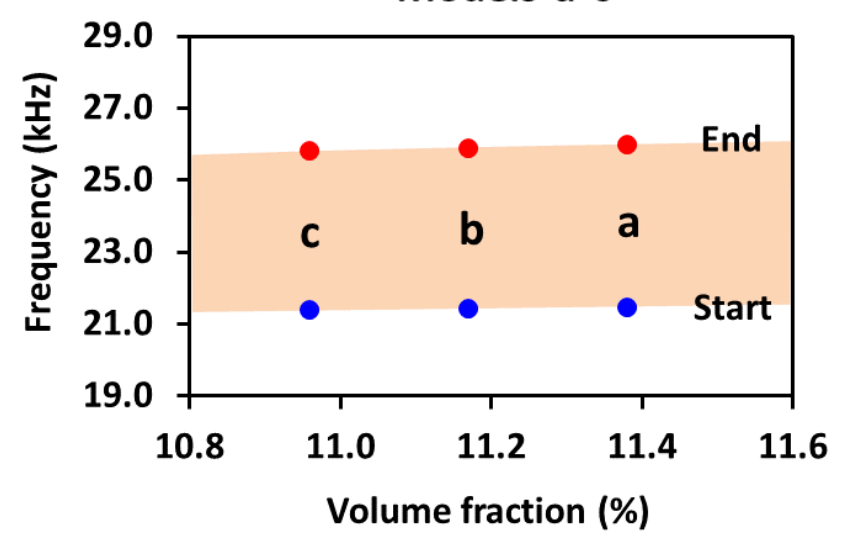

(a)
Models d-f

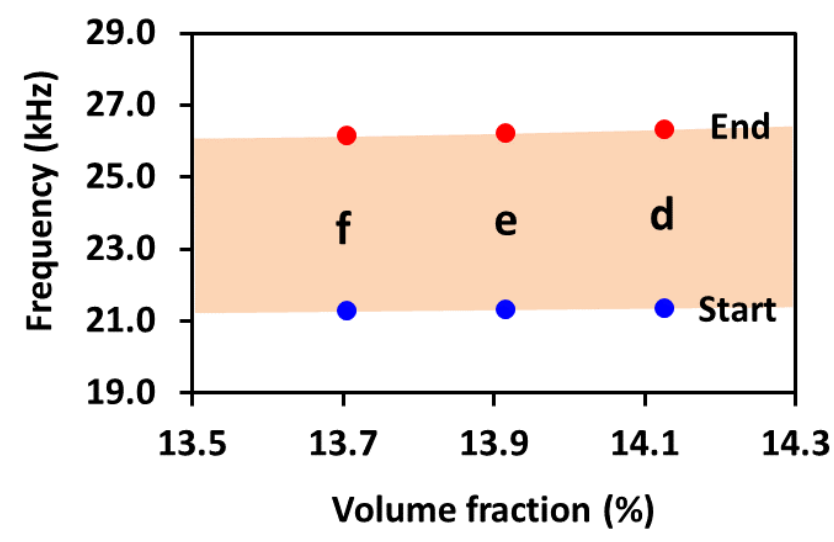

(b)

Fig. 11 Shift in start and end of the bandgap along X-M due to the change in volume fraction 
In Figs. 9-11, it can be seen the influence of the resonator's volume fraction on stop bandwidth. However, because of the different scaling in both $\mathrm{x}$ - and $\mathrm{y}$-axis it can be a little difficult to identify how much the volume fraction is taking part in controlling the bandgap width for various situations. For example, in Figs. 9-10, all the presentations look almost the same. In that context, the volume fraction possesses almost equal influence for both the set of models and for both overall and $\mathrm{x}$ directional waves. To have a clearer understanding, a percentage increase in bandgap width due to the increase of volume fraction is plotted in Fig. 12. It can be seen that using the model set-1, maximum increase (about 135\%) can be obtained in the case of overall bandwidth (ref. Fig. 12(a)). Whereas, almost negligible increase is visible in X-M directional wave. A similar trend can be visible for model set-2 as well (ref. Fig. 12(b)). However, the maximum increase for set- 2 in the overall bandgap is about $50 \%$, which is significantly lower than the gain reported for set-1 in Fig. 12(a).

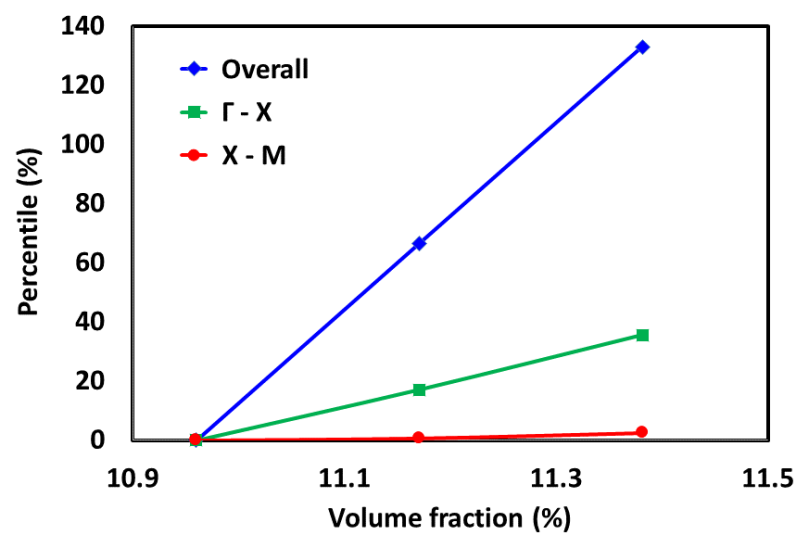

(a)

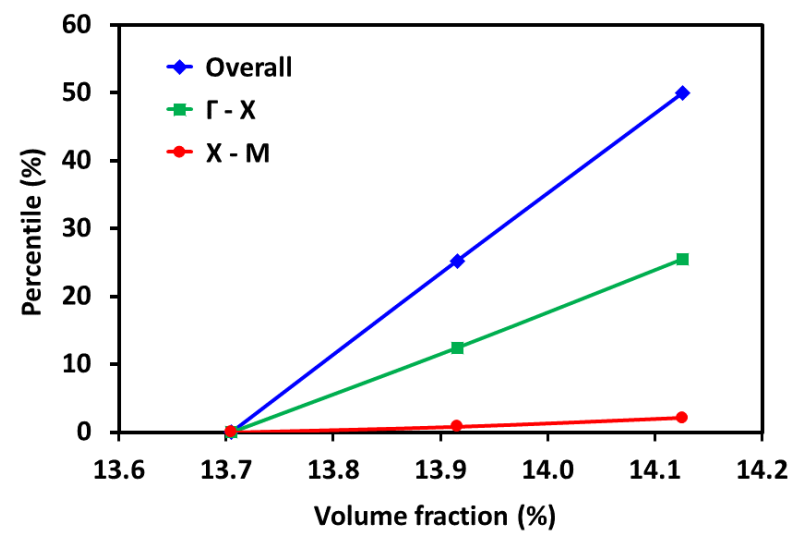

(b)

Fig. 12 Percentage increase in band gap width for models (a) 1a-1c (set 1), (b) 1d-1f (set 2)

\section{Conclusion}

Manipulating frequency band gaps by designing novel configurations of metamaterials is one of the key areas of interest in recent days. In this purpose, mass-in-mass and layer-in-layer models were proposed by the previous researchers. However, dependency of frequency band gaps on resonator setting and volume fraction of the resonators in a unit cell were still to investigate. In this work, multi-scale mass-in-mass systems containing split-ring resonators are investigated to understand the influence of resonator setting and volume fraction on the frequency stop bands. It has been found that, in any resonating setup, width of the frequency bandgap can be linearly manipulated through the alteration of volume fraction of resonators in the unit cell. However, the linear pattern can be shifted sidewise by changing the resonator set by introducing or taking off a resonator from the unit cell. It has been noticed that lower band of the stop band remains unaffected with the change of volume fraction and the upper band can be shifted upward/downward through the increase/decrease of volume fraction of the resonators. This study also confirms that, with a lower number of resonators it is possible to manipulate the frequency band gap heavily by changing the volume fraction, in comparison to a higher number of resonators. In totality, this study concludes that, in a metamaterial, frequency bands are strongly sensitive to resonator setting and volume fraction in a unit cell.

\section{Acknowledgement}

This research was partially funded by the Office of Vice President of Research, University of South Carolina.

\section{References}

[1] Smith, D.R., Pendry, J.B. and Wiltshire, M.C., 2004. Metamaterials and negative refractive index. Science, 305(5685), pp.788-792.

[2] Pendry, J.B., Holden, A.J., Robbins, D.J. and Stewart, W.J., 1999. Magnetism from conductors and enhanced nonlinear phenomena. IEEE transactions on microwave theory and techniques, 47(11), pp.2075-2084.

[3] Smirnova, E.I., Mastovsky, I., Shapiro, M.A., Temkin, R.J., Earley, L.M. and Edwards, R.L., 2005. Fabrication and cold test of photonic band gap resonators and accelerator structures. Physical Review Special TopicsAccelerators and Beams, 8(9), p.091302.

[4] Liu, Z., Chan, C.T. and Sheng, P., 2005. Analytic model of phononic crystals with local resonances. Physical Review B, 71(1), p.014103.

[5] Mei, J., Liu, Z., Wen, W. and Sheng, P., 2006. Effective mass density of fluid-solid composites. Physical review letters, 96(2), p.024301.

[6] Ahmed, R. and Banerjee, S., 2013, April. Novel split ring metamaterial for multiple band gaps and vibration control. In Health Monitoring of Structural and Biological Systems 2013 (Vol. 8695, p. 86952L). International Society for Optics and Photonics.

[7] Ahmed, H., Ahmed, R., Indaleeb, M.M. and Banerjee, S., 2018. Multifunction acoustic modulation by a multi-mode acoustic metamaterial architecture. Journal of Physics Communications, 2(11), p.115001.

[8] Sigalas, M.M. and Economou, E.N., 1992. Elastic and acoustic wave band structure. Journal of sound and vibration, 158(2), pp.377-382.

[9] Poulton, C.G., Movchan, A.B., McPhedran, R.C., Nicorovici, N.A. and Antipov, Y.A., 2000. Eigenvalue problems for doubly periodic elastic structures and phononic band gaps. Proceedings of the Royal Society of London. Series A: Mathematical, Physical and Engineering Sciences, 456(2002), pp.2543-2559. 
[10] Ahmed, R. and Banerjee, S., 2015. Bio-Inspired Design of a Multi-scale Pass Band Frequency Sensor Using Local Resonance Phenomena. In Experimental and Applied Mechanics, Volume 6 (pp. 21-27). Springer, Cham.

[11] Ahmed, R., Madisetti, D. and Banerjee, S., 2017. A subwavelength scale acoustoelastic sonic crystal for harvesting energies at very low frequencies $(<\sim 1 \mathrm{kHz})$ using controlled geometric configurations. Journal of Intelligent Material Systems and Structures, 28(3), pp.381-391.

[12] Banerjee, S. and Ahmed, R., University of South Carolina, 2020. Power optimization for a unit cell metamaterial energy harvester. U.S. Patent 10,694,466.

[13] Hirsekorn, M., Delsanto, P.P., Batra, N.K. and Matic, P., 2004. Modelling and simulation of acoustic wave propagation in locally resonant sonic materials. Ultrasonics, 42(1-9), pp.231-235.

[14] Oudich, M., Li, Y., Assouar, B.M. and Hou, Z., 2010. A sonic band gap based on the locally resonant phononic plates with stubs. New Journal of Physics, 12(8), p.083049.

[15] Caballero, D., Sánchez-Dehesa, J., Rubio, C., MartinezSala, R., Sánchez-Pérez, J.V., Meseguer, F. and Llinares, J., 1999. Large two-dimensional sonic band gaps. Physical Review E, 60(6), p.R6316.

[16] Chesnais, C., Boutin, C. and Hans, S., 2012. Effects of the local resonance on the wave propagation in periodic frame structures: Generalized Newtonian mechanics. The Journal of the Acoustical Society of America, 132(4), pp.2873-2886.

[17] Ahmed, R.U. and Banerjee, S., 2013. Wave propagation in metamaterial using multiscale resonators by creating local anisotropy. International Journal of Modern Engineering, 13(2), p.51.

[18] Liang, X., Wang, T., Jiang, X., Liu, Z., Ruan, Y. and Deng, Y., 2019. A numerical method for flexural vibration band gaps in a phononic crystal beam with locally resonant oscillators. Crystals, 9(6), p.293.
[19] J LUO, J., YAO, L., JIANG, G. and WU, F., 2019. A study on the vibration band gap and vibration characteristics of a cylindrical shell phononic crystal. Journal of Vibration and Shock, (8), p.20.

[20] Huang, G.L. and Sun, C.T., 2010. Band gaps in a multiresonator acoustic metamaterial. Journal of Vibration and Acoustics, 132(3).

[21] Huang, H.H., Sun, C.T. and Huang, G.L., 2009. On the negative effective mass density in acoustic metamaterials. International Journal of Engineering Science, 47(4), pp.610-617.

[22] Ahmed, R.U. and Banerjee, S., 2013. Wave propagation in metamaterial using multiscale resonators by creating local anisotropy. International Journal of Modern Engineering, 13(2), p.51.

[23] Movchan, A.B. and Guenneau, S., 2004. Split-ring resonators and localized modes. Physical Review B, 70(12), p.125116.

[24] Hsu, J.C., 2011. Local resonances-induced low-frequency band gaps in two-dimensional phononic crystal slabs with periodic stepped resonators. Journal of Physics D: Applied Physics, 44(5), p.055401.

[25] Liu, Z., Zhang, X., Mao, Y., Zhu, Y.Y., Yang, Z., Chan, C.T. and Sheng, P., 2000. Locally resonant sonic materials. Science, 289(5485), pp.1734-1736.

[26] Li, J. and Chan, C.T., 2004. Double-negative acoustic metamaterial. Physical Review E, 70(5), p.055602.

[27] Milton, G.W. and Willis, J.R., 2007. On modifications of Newton's second law and linear continuum elastodynamics. Proceedings of the Royal Society A: Mathematical, Physical and Engineering Sciences, 463(2079), pp.855-880.

[28] Yao, S., Zhou, X. and Hu, G., 2008. Experimental study on negative effective mass in a $1 \mathrm{D}$ mass-spring system. New Journal of Physics, 10(4), p.043020. 